\title{
Efeitos do isolamento na pandemia por COVID-19 no comportamento de crianças e adolescentes com autismo
}

\section{Effects of isolation in COVID-19 pandemic on the behavior of autistic children and adolescents}

\author{
Rosana Carla do Nascimento Givigi*1 \\ Raquel Souza Silva*2 \\ Edênia da Cunha Menezes*3 \\ João Rafael Santos Santana*4 \\ Clayne Mirelle Pereira Teixeira*5
}

\begin{abstract}
Com a pandemia da COVID-19 a Organização Mundial da Saúde declarou estado de emergência de saúde pública. O isolamento tem transformado as relações sociais de crianças e adolescentes com Transtorno do Espectro do Autismo. Este estudo objetivou analisar os efeitos do isolamento no comportamento desses sujeitos. Trata-se de um estudo descritivo, de caráter transversal, no qual pais ou responsáveis responderam um questionário online. Os resultados da pesquisa apontaram que o confinamento acentuou os sintomas do autismo. Conclui-se que são necessárias medidas de intervenções com os sujeitos e suas famílias.
\end{abstract}

Palavras-chave: Transtorno autístico, comportamento, COVID-19, isolamento social

*1, 2, 4,5 Universidade Federal de Sergipe - UFS (Aracaju, SE, Brasil).

*33 New York University (New York, NY, E.U.A.). 


\section{Introdução}

A pandemia da COVID-19 mobilizou pesquisadores de diversas partes do mundo em várias frentes científicas. Numa situação de Emergência de Saúde Pública, desde o início do ano de 2020, a Organização Mundial da Saúde (OMS) declarou a gravidade da doença causada pelo novo coronavírus (SARS-CoV-2), caracterizada como uma pandemia. Os dados de setembro de 2021 confirmam mais de 223 milhões de casos de COVID-19 e 4,6 milhões mortes no mundo, sendo que no Brasil são quase 21 milhões de casos confirmados e mais de 585 mil mortos (World Health Organization, 2021; Brasil, 2021c).

A gravidade da situação produziu um impacto mundial em diversos aspectos como o econômico, social, de saúde, educacional, dentre outros. No Brasil, para o combate à COVID-19, a União gastou 524 bilhões de reais em 2020, estando previstos mais 137,2 bilhões de reais em 2021 (Brasil, 2021a). Mesmo assim o Brasil se tornou um polo de disseminação do vírus da COVID-19 e continua tendo dificuldade no controle da pandemia. Os motivos apontados são: a contrapropaganda da Presidência da República; a ação ineficaz dos gestores públicos; a baixa testagem da população; a baixa adesão da população às medidas de isolamento social, dentre outros (Aquino, E.M., Silveira, Pescarini, Aquino \& Souza-Filho, 2020).

A situação causada pela pandemia tem provocado diferentes impactos na vida das pessoas. As famílias que em sua constituição têm como membro pessoas com deficiência vivem situações particulares, conforme a gravidade do caso. Para ter uma noção de como a pandemia está afetando as pessoas com autismo no Brasil é necessário compreender o transtorno e suas consequências.

Atualmente entende-se que o autismo se apresenta de forma e intensidade distintas, por isso chamado de Transtorno do Espectro Autista (TEA). O Manual Diagnóstico e Estatístico de Transtornos 
Mentais da Associação Psiquiátrica Americana (DSM-5) caracteriza-o como um transtorno invasivo do desenvolvimento, com três grandes áreas de comprometimento: interação social; comunicação verbal; e comportamento, com interesses restritos e movimentos repetitivos (estereotipias). Tendo um conjunto muito variado de sintomas, manifesta-se antes dos três anos de idade (Amaral \& Vries, 2020; Santos \& Lemes, 2020; American Psychiatric Association, 2013; Viana, Furtado \& Vieira, 2020).

Em relação ao comportamento de crianças e adolescentes com autismo, é habitual encontrar na literatura ou escutar de pais, professores e profissionais que eles apresentam déficit na área social, marcada fortemente por dificuldade de interação, interesses restritos e movimentos repetitivos, agressividade, dificuldade em mudança de rotina, alteração de sensibilidade, impulsividade e agitação, distúrbios do sono, choro excessivo, birras de difícil controle, ansiedade, dentre outros (Visani \& Rabello, 2012; Narzisi, 2020; Amaral \& Vries, 2020; Briet et al., 2017).

Se pessoas com autismo têm dificuldades de interação, o isolamento social poderá ser mais prejudicial a essas pessoas. Um artigo publicado recentemente na Austrália, sobre adultos com autismo, relatou que o distanciamento social, que foi adotado como medida para controle da COVID-19, pode trazer consequências significativas para a saúde mental das pessoas com autismo (Houring, 2020), pois elas já têm uma probabilidade maior de sofrer de doença mental (Cjevic, Arnold, Foley \& Trollor, 2018).

As crianças e adolescentes com TEA também estão sujeitas aos efeitos do isolamento social que, como uma das medidas para conter a pandemia, resultou no fechamento de escolas, de eventos públicos e privados, de parques, de serviços terapêuticos e de reabilitação, instituindo a quarentena obrigatória para pessoas infectadas e não infectadas (Lewnard \& Lo, 2020).

Pesquisadores de diferentes países incluíram em seus estudos como as pessoas com autismo estavam reagindo à pandemia, e constatou-se que crianças com TEA apresentavam maior risco de sentirem-se frustradas, angustiadas, ansiosas, estressadas, podendo agravar os problemas comportamentais (Amaral \& Vries, 2020; Palacio-Ortiz et al., 2020; Colizzi et al., 2020).

Com as rotinas duramente modificadas e com as medidas de isolamento impostas às famílias, as crianças precisaram limitar a brincadeira e o estudo para dentro de casa, bem como interromper tratamentos e evitar contato social com os pares. No Brasil, registram-se poucos trabalhos de pesquisa especificamente com crianças e adolescentes com autismo nesse período pandêmico (Barbosa et al., 2020; Fernandes et al., 2020). Dessa forma, o objetivo desta 


\section{ARTIGOS}

pesquisa foi analisar os efeitos do isolamento no comportamento de crianças e adolescentes com autismo.

\section{Metodologia}

Trata-se de um estudo descritivo, de caráter transversal. Este estudo foi aprovado pelo Comitê de Ética e Pesquisa (CEP) envolvendo seres humanos da Universidade Federal de Sergipe, n. 33464320.9.0000.5546.

\section{Formato da pesquisa}

O questionário foi feito a partir do Formulário Google e compartilhado por meio da divulgação de um hiperlink. A pesquisa ficou disponível online de 3 de julho a 11 de agosto de 2020. Todos os participantes assinaram o termo de consentimento, no próprio formulário eletrônico, que continha informações sobre o objetivo do estudo, seus procedimentos, sobre a participação voluntária e informações de contato dos pesquisadores.

O questionário tinha 21 questões, sendo 19 assertivas (seis de resposta única, cinco dicotômicas, oito questões de múltipla escolha, e duas de resposta aberta) divididas em três categorias: (a) as características sociodemográficas e os aspectos psicossociais; (b) o impacto do isolamento, provocado pela pandemia da COVID-19, no comportamento da criança ou adolescente com autismo; e (c) o impacto do isolamento, provocado pela pandemia da COVID-19, na linguagem e na comunicação da criança ou adolescente com autismo. As categorías a e b farão parte deste artigo.

$\mathrm{Na}$ categoría a, características sociodemográficas e os aspectos psicossociais, foram 17 questões com os seguintes conteúdos: sexo, idade, renda da família; número de pessoas que moravam na casa; se os moradores estavam ficando em casa durante a quarentena; que atividades as crianças e adolescentes faziam antes e durante a quarentena; quais atividades continuavam virtualmente; quais atividades de lazer eram habituais nos fins de semana antes e durante a quarentena; se a criança e o adolescente pediam para sair. Das 17 questões apenas duas eram abertas: a que interrogava a idade e a que perguntava quem estava saindo na casa. Todas as outras questões eram assertivas, sendo sete de resposta única, seis de múltipla escolha e duas dicotômicas. 
$\mathrm{Na}$ categoría $\mathrm{b}$, o impacto do isolamento provocado pela pandemia da COVID-19 no comportamento da criança ou adolescente com autismo, foram três questões: se a criança/adolescente mudou o comportamento durante o isolamento; se as mudanças foram positivas ou negativas; e, por último, havia uma lista com 12 comportamentos listados (agitado, tranquilo, birrento, triste, feliz, sonolento, sem sono, com mais movimentos repetitivos, mais interativo, isolando-se mais, brincando melhor, expresando-se melhor) em que podiam ser marcadas quantas alternativas a criança/adolescente se encaixasse.

\section{Participantes}

Os participantes foram pais ou responsáveis, ou profissionais autorizados pelos pais de crianças e adolescentes de dois a 19 anos com autismo. O contato com os participantes foi feito a partir de listas e grupos disponíveis em sites de associações e redes sociais. O diagnóstico de TEA foi por autodeclaração.

\section{Análise dos dados}

\section{Descrição das variáveis}

Os dados foram analisados de forma que fizesse a caracterização dos participantes, a caracterização sociodemográfica e dos aspectos psicossociais, bem como efeitos do isolamento para o comportamento da criança e adolescente com autismo, no período da pandemia da COVID-19. As variáveis analisadas foram: perfil dos respondentes do questionário; gênero e idade cronológica das crianças e adolescentes com autismo; atividades das crianças e adolescentes com autismo no período pré-pandêmico; atividades que tiveram continuidade no período pandêmico; atividades que a criança ou adolescente tem feito em casa durante o isolamento; alterações de comportamento durante o isolamento.

\section{Análise estatística}

Os dados brutos foram baixados do Formulário Google para um arquivo de planilha de dados do programa Excel for Windows 2013. Para análise dos dados foi realizada descrição do perfil da amostra segundo as variáveis do estudo. Depois da classificação das variáveis, a análise de conteúdo foi 


\section{ARTIGOS}

realizada no software MAXQDA para classificar definição, atributos, similaridades e diferenças entre as variáveis. Os valores foram representados em mediana e intervalo interquartil.

\section{Resultados}

Participaram da pesquisa 322 pais ou responsáveis. Os resultados serão apresentados a seguir:

\section{Características sociodemográficas e os aspectos psicossociais}

O total de questionários submetidos foram 322, dos quais 263 foram respondidos pelas mães das crianças ou dos adolescentes, representando $81,7 \%$, seguido por 30 pais, o que representou 9,3\% (Tabela 1 ).

\section{Tabela 1}

Grau de parentesco ou proximidade com o menor

\begin{tabular}{l|c}
\hline Respondente & Número \\
\hline Avó ou avô & 08 \\
\hline Irmão & 01 \\
\hline Madrinha & 11 \\
\hline Mãe & 263 \\
\hline Pai & 30 \\
\hline Professora & 03 \\
\hline Tia ou tio & 06 \\
\hline TOTAL & $\mathbf{3 2 2}$ \\
\hline
\end{tabular}


O perfil de gênero correspondeu a 79,8\% (257) do sexo masculino, 20,2\% (65) do sexo feminino. As idades variaram de dois a 19 anos, sendo que a maior quantidade $78,3 \%$ (252) encontrava-se entre três a 11 anos. A Tabela 2, com a faixa etária dos participantes, apresenta uma mediana igual a sete anos de idade e uma média aritmética igual a 7,5 (ou seja, sete anos e meio), (64,74 (6-6) $n=252$ ).

Tabela 2

Idade cronológica das crianças e adolescentes com autismo

\begin{tabular}{|c|c|}
\hline Idade (anos) & Quantidade (n) \\
\hline 2 & 17 \\
\hline 3 & 33 \\
\hline 4 & 44 \\
\hline 5 & 27 \\
\hline 6 & 32 \\
\hline 7 & 29 \\
\hline 8 & 27 \\
\hline 9 & 24 \\
\hline 10 & 15 \\
\hline 11 & 21 \\
\hline 12 & 10 \\
\hline 13 & 12 \\
\hline 14 & 11 \\
\hline 15 & 5 \\
\hline 16 & 4 \\
\hline 17 & 5 \\
\hline 18 & 5 \\
\hline 19 & 1 \\
\hline
\end{tabular}




\section{ARTIGOS}

Todas as regiões do Brasil foram representadas, sendo $9(2,8 \%)$ da região Centro-Oeste, $61(18,9 \%)$ do Sudeste, $31(9,6 \%)$ do Sul, $11(3,4 \%)$ do Norte e $210(65,2 \%)$ do Nordeste, o maior número de respondentes. Dos 27 estados brasileiros, 19 foram representados na pesquisa.

Um total de 74,2\% (239) das famílias tinha renda de até 5 mil reais, merecendo destaque o fato de $22,7 \%$ (52) delas ganharem até 1 salário mínimo. Porém, durante o período pandêmico $55,7 \%$ (179) das famílias tiveram sua renda diminuída. Outro dado é que 73,6\% (237) famílias eram formadas por 3 ou 4 membros. Apenas 4\% (13) das crianças e adolescentes estavam saindo durante a quarentena, e em 69\% (222) das famílias um dos adultos saía com frequência.

Analisando a rotina anterior dessas crianças e adolescentes, constatou-se que dos 322 participantes 281 estudavam em escola regular e $30 \mathrm{em}$ escola especial, portanto, $96,6 \%$ estudavam, o que significa que ficavam pelo menos um turno diário fora de casa. Além disso, $100 \%$ das crianças e adolescentes faziam outras atividades semanais, dentre elas as esportivas, as religiosas, de artes e terapêuticas. Dentre as atividades realizadas, um número expressivo (148 crianças e adolescentes) fazia atividades esportivas. Como suportes terapêuticos, 62,4\% (201) faziam terapia ocupacional, 71,7\% (230) terapia fonoaudiológica, e 70,2\% (226) acompanhamento psicológico.

Durante o período pandêmico os atendimentos terapêuticos tiveram grande queda, conservando-se de forma virtual apenas 30 dos 201 (14,9\%), 30 dos $230(13 \%)$ e 30 dos $226(13,3 \%)$, respectivamente. Quanto às atividades, todas foram quantificadas; uma mesma criança ou adolescente poderia estar executando várias atividades. O total de atividades foi de 1251 no período pré-pandêmico, e caiu para 391 durante a pandemia. Constatou-se que apenas $31,2 \%$ das atividades permaneceram durante o período pandêmico. Como exemplo citamos as atividades esportivas que caíram de 148 para 8, portanto, apenas $5,4 \%$ dos sujeitos.

Dentre as atividades de lazer que eram habituais nos fins de semana ou feriados, as mais frequentes foram: ir à casa de parentes ou amigos (235), ir à praia (117), ir ao parquinho (183), ir ao shopping (149), e brincar na rua (151). Porém, com o isolamento 168 (52,3\%) crianças e adolescentes deixaram de participar dessas atividades de lazer, ficando sem elas ou substituindo-as por assistir televisão, brincar dentro de casa, brincar no quintal, e jogar bola. 


\section{O impacto do isolamento}

Com a restrição de atividades, na opinião de pais ou responsáveis, apenas 9\% (29) das crianças e adolescentes não estavam incomodadas, 37\% (119) estavam um pouco incomodadas pedindo um pouco para sair, e 54\% (174) estavam muito incomodadas pedindo muito para sair.

Quando interrogados sobre o que as crianças e adolescentes estavam fazendo durante o isolamento, houve uma grande variedade de respostas (Tabela 3), destacando-se assistir televisão, brincar sozinho ou com adultos, jogar no tablet ou celular e desenhar.

\section{Tabela 3}

\section{Atividades que a criança ou adolescente faziam em casa durante o isolamento}

\begin{tabular}{l|c}
\hline \multicolumn{1}{c|}{ Ocupações de casa } & Número de crianças e adolescentes \\
\hline Ajudado em tarefas domésticas & 81 \\
\hline Andando de bicicleta ou patinete & 55 \\
\hline Assistindo televisão & 264 \\
\hline Brincando com adultos & 194 \\
\hline Brincando com outras crianças & 69 \\
\hline Brincando sozinho & 211 \\
\hline Desenhando & 147 \\
\hline Jogando no celular ou tablet & 253 \\
\hline $\begin{array}{l}\text { Tem ficado grande parte } \\
\text { do dia sem atividades }\end{array}$ & 45 \\
\hline Tem lido ou escutado estórias & 107 \\
\hline
\end{tabular}




\section{ARTIGOS}

No que se refere ao comportamento, 87,3\% (281) das famílias afirmaram que a criança/adolescente mudou o comportamento durante o isolamento. Quando interrogados se a mudança era positiva ou negativa, 68,6\% (221) avaliaram que as mudanças foram negativas, e 31,4\% (101) que foram positivas.

Em relação ao comportamento, poderiam ser apontadas mais de uma das alternativas. Desta forma, as principais mudanças negativas apontadas no comportamento foram: 33\% (73) dos participantes disseram que era ficar isolado, e 54\% (119) disseram reagir negativamente à interação. Dentre os 101 participantes que disseram que as mudanças de comportamento foram positivas, foram destaque: brincar melhor (32 participantes, 31,6\%) e expressar-se melhor (46 participantes, 46\%).

\section{Discussão}

Os questionários foram em sua maioria respondidos pelas mães e a maioria das crianças ou adolescentes da pesquisa são do sexo masculino. Estudos como os de Cunha, Santos e Carlos (2016), Lima e Couto (2020), também tiveram as mães como responsáveis pela participação nas pesquisas de crianças com autismo. Este é um cenário comum quando se trata dos cuidados aos filhos, especialmente quando estes possuem alguma deficiência. Historicamente a prática do cuidado está relacionada ao feminino, uma premissa que foi sustentada na sociedade por regulações religiosas, valores e acordos morais (Leonardo \& Morais, 2017; Dias et al., 2020; Rooke et al., 2019).

Outro dado que coaduna com a literatura é a grande maioria das crianças e adolescentes serem do sexo masculino. Estudos que descreveram as características ou perfil epidemiológico de pessoas com autismo também encontraram valores semelhantes. É reconhecida pela literatura a predominância do autismo em pessoas do sexo masculino (Lima et al., 2019; Knutsen, et al., 2019).

Quanto ao perfil socioeconômico a maioria das famílias encontra-se nas classes C, D e E, o que é esperado, já que no Brasil apenas 15\% da população faz parte das classes A e B (IBGE, 2018). Preocupante é o número de famílias que recebe até 1 salário mínimo: 22.7\%. Provavelmente uma parte dessas famílias vive com o Benefício de Prestação Continuada (BPC) pago pelo Governo Federal. Esse recurso está previsto nas formas de amparo a pessoa com 
deficiência, que, no Brasil, está na Lei Orgânica da Assistência Social (LOAS), Decreto-Lei n. 8.742 (Brasil, 1993) e no Decreto-Lei n. 6.214 (Brasil, 2007).

Analisando os impactos provocados pelo isolamento, resultado da pandemia da COVID-19, um pouco mais da metade dessas famílias teve sua renda diminuída nesse período, o que já irá provocar mudanças. Considerando que apenas $4 \%$ das crianças e adolescentes com autismo têm saído durante a quarentena, entende-se que modificaram radicalmente suas rotinas já que anteriormente $96,6 \%$ deles estudavam. Além disso, todos faziam algum tipo de atividade (artística, esportiva e religiosa) semanalmente e/ou algum tipo de terapia, aumentando ainda mais o tempo que passavam fora de casa. Importante destacar que, ao ficarem sem as terapias, as crianças e adolescentes deixavam de ser apoiadas por um profissional, o que inevitavelmente aumentava a sobrecarga das famílias (Narzisi, 2020).

$\mathrm{Na}$ rotina normal, antes da pandemia, a família passava várias horas do dia sem ter obrigações diretas com seus filhos, dividindo não somente o trabalho, mas as ansiedades, dúvidas e problemas. Essas mudanças de rotina podem causar à pessoa com autismo um grande sofrimento. Por essa razão, os pais devem estabelecer novas rotinas, de maneira que a criança e o adolescente sintam-se seguros e apoiados (Narzisi, 2020).

Outro fator alterado foi o lazer, sendo as atividades habituais como ir à praia ou ao parque, substituídas por atividades feitas dentro de casa, como assistir televisão ou brincar.

Em relação ao comportamento, a grande maioria das famílias constatou que seus filhos ficavam incomodados em ficar em casa e pediam para sair. A mudança de rotina habitualmente provoca ansiedade e desconforto em crianças com autismo. Também constataram que seus filhos tiveram mudanças de comportamento; $68,6 \%$ avaliaram que as mudanças foram negativas. $\mathrm{O}$ fato de as mudanças do comportamento serem negativas alerta-nos para os modos que essas crianças e adolescentes estão sendo apoiados. A pessoa com autismo enfrenta ao longo da vida diferentes desafios de comunicação social, de comportamento, e de funcionamento adaptativo. A gravidade dessas dificuldades varia entre os indivíduos, formando quadros heterogêneos, exigindo diferentes modos de intervenção (Ameis et al., 2020).

Pesquisas com crianças com autismo na Itália, Turquia, Estados Unidos, no período de pandemia da COVID-19, mostraram que as famílias sentiram-se desamparadas, tiveram um aumento de estresse e ansiedade por estarem sozinhas na tarefa de regular todas as atividades da criança e adolescente com autismo (Colizzi et al., 2020; Ersoy et al., 2020). 


\section{ARTIGOS}

Uma solução encontrada nas áreas de saúde e educacional para minimizar os problemas foi o teleatendimento ou intervenção à distância. Inegavelmente, esse tipo de serviço requer uma preparação específica tanto no uso da tecnologia, quanto a adaptação de objetivos e conteúdos. Porém, a maioria dos estados brasileiros não estava preparada para esse tipo de atendimento. Em alguns países como Estados Unidos, Reino Unido, Bélgica, Alemanha, já se fazia intervenção através de programas online de reabilitação utilizando o computador sem a ação presencial de um profissional (Halim et al., 2020; Amaral \& Vries, 2020).

No Brasil, as práticas de intervenção online não eram comuns até a pandemia, e vale ressaltar que não houve tempo para treinamento. Os profissionais de saúde e de educação tiveram que aprender maneiras de interagir de forma remota, mas no caso do atendimento a pessoas com autismo o desafio foi ainda maior. Para que uma aula ou atendimento possa utilizar determinados recursos tecnológicos são necessárias boas plataformas online; porém, em virtude da realidade econômica e todas as questões macrossociais atuais do país, a grande maioria das famílias não tem acesso a essas plataformas e a bons recursos tecnológicos que possam garantir a eficácia de suporte e orientação (Farias \& Leite, 2020; Fernandes et al., 2020). Essa é a realidade em muitos países da América Latina: a dificuldade de obter os dispositivos tecnológicos e de ter acesso a internet de qualidade (Rattazzi, 2020).

Chama atenção que as principais questões negativas em relação ao comportamento estejam relacionadas à interação, com comportamentos como isolar-se e evitar o outro. Em pesquisas feitas em outros países constatou-se que no período de isolamento os pais ou cuidadores de crianças com autismo relataram que seus filhos estavam menos cooperativos, com mais estereotipias, mais irritados, com mais dificuldades de interação (Degli Espinosa et al., 2020).

O fato de uma criança se isolar, quase sempre traz incômodo aos pais. É preciso compreender que no caso da criança com autismo não se refere ao silêncio que faz parte do jogo intersubjetivo da comunicação, mas sim como manifestação de proteção por suas dificuldades de relação (Zornig, 2019).

O questionário não trata especificamente da relação entre os comportamentos negativos e a interrupção das terapias, mas, como foi descrito, menos de $15 \%$ dos tratamentos - como a terapia ocupacional, a fonoaudiologia e a psicologia - foram mantidos; o não acompanhamento terapêutico pode ter aumentado as dificuldades de interação e o isolamento. Esta é apenas uma hipótese, já que o questionário não ampliou essas relações, sendo essa uma limitação percebida após os resultados. 
Estudos de pesquisadores de vários países mostraram que as questões de saúde mental merecem cuidados específicos nesses tempos de isolamento. A situação de distanciamento provocou uma série de movimentos por parte do Ministério da Saúde, de Conselhos Federais como de Medicina, Psicologia, Fonoaudiologia e Enfermagem, o que fez emergir regulamentações, diretrizes e orientações sobre os cuidados com a saúde mental nesse momento específico (Lima, 2020; Conselho Federal de Medicina, 2020; Conselho Federal de Psicologia, 2020; Brasil, 2020).

Os cuidados devem ser redobrados quando se fala da população infanto-juvenil, e mais ainda quando essa população faz parte do grupo de pessoas em situação de vulnerabilidade, como é o caso do autismo (Lima, 2020; Laboratório de Pesquisa em Saúde Mental e Terapia Ocupacional da UFSCAR, 2020). A mudança de rotina, a necessidade de suporte escolar e terapêutico, a dificuldade que as pessoas com autismo têm de processar as mudanças, a sobrecarga das famílias em assumir o cuidado e as demais obrigações, a diminuição do número de parceiros interacionais, especialmente da mesma faixa etária, a falta de entendimento do momento de isolamento que pode causar um aumento de ansiedade, são apontadas como razões pelos aspectos negativos do comportamento segundo os responsáveis (Barbosa et al., 2020).

Aproximadamente $1 / 3$ dos respondentes avaliaram que as mudanças de comportamento foram positivas, com destaque para o brincar e se expressar melhor. Estudos em outros países atribuíram que ficar tempo integral em casa pode melhorar o comportamento de algumas crianças com autismo pelas seguintes razões: o ambiente doméstico aceita uma programação individualizada e por isso diminui a ansiedade; há uma diminuição das distrações; estarão expostos a menos conflitos; menos estímulos sensoriais; maior tempo com a família; atividades mais calmas; dentre outras (Amaral \& Vries, 2020).

Aprofundando a discussão sobre as mudanças positivas no comportamento de crianças e adolescentes com autismo durante o isolamento, outras justificativas foram encontradas, como: crianças com irmãos ficam melhores em casa durante o isolamento; famílias que têm apoio entre seus membros enfrentam melhor o isolamento; capacidade de adaptação dos pais; flexibilidade na criação de atividades para os filhos (Cahapay, 2020, Guidotti et al., 2020).

Em pesquisas feitas nos Estados Unidos e Itália, outro fator apontado que contribuiria para que na pandemia as crianças e adolescentes apresentassem mudanças positivas seria a melhor condição financeira, porque essas famílias poderiam promover melhor bem-estar para seus filhos (Ameis et al., 2020; Eshraghi et al., 2020). Porém, nesta pesquisa, fazendo um cruzamento 


\section{ARTIGOS}

dos dados entre a realidade socioeconômica e as mudanças positivas no comportamento das crianças e adolescentes com autismo percebe-se que não houve relação entre esses fatores. Neste estudo constata-se que o fato de ter mais recursos financeiros não garante que crianças e adolescentes com autismo estejam bem em situações de agravamento como a provocada pela pandemia.

Analisa-se que quanto melhor for a condição da família em lidar com o conjunto de questões problemáticas na comunicação social, no comportamento, na interação, na estruturação da rotina, dentre outras, mais chances as crianças e adolescentes com autismo terão na superação das dificuldades (Colizzi et al., 2020).

Os resultados deste estudo coadunam com outros estudos com temáticas semelhantes como os de Araújo e Fernandes (2020), Narzisi (2020), Ameis et al. (2020), Kawabe et al. (2020), que apontam para a necessidade de criação de medidas de intervenções em teleatendimento; orientações às famílias; apoio às famílias na preparação e garantia de rotinas habituais e alternativas; regulação das atividades que envolvem aparelhos eletrônicos como tablets, celulares, videogames e televisão; dentre outras.

\section{Considerações finais}

Os resultados da pesquisa apontam que o confinamento provocado pela pandemia da COVID-19 acentuou os sintomas de crianças e adolescentes com autismo. Após análises, pode-se enumerar algumas evidências: 1) Neste estudo, apenas a desvantagem econômica e social parece não ter tornado o impacto da pandemia mais danoso, porém isto pode ser resultado de uma limitação de acesso à internet das pessoas mais pobres, ou mesmo outras limitações para responder o questionário online; 2) crianças e adolescentes com autismo necessitam de intervenções profissionais, e por isso a interrupção dos tratamentos coloca a família em situação de fragilidade; 3) a pandemia mostrou o quanto as políticas intersetoriais no Brasil não dialogam para oferecer suporte em situações como essa; 4) o Brasil não estava preparado para o teleatendimento; 5) é importante a criação de redes de apoio para as famílias das crianças e adolescentes com autismo; 6) ainda há necessidade de ações informativas sobre o cuidado; 7) é necessário compreender as particularidades vividas pelas crianças e adolescentes com autismo na situação de isolamento social; 8) no cenário pandêmico a maioria das crianças 
e adolescentes com autismo foram impactadas negativamente em relação ao comportamento.

Dentre as limitações do presente estudo estão questões como: relação entre idades das crianças e efeitos da pandemia; a possibilidade da ocorrência de viés nas respostas a algumas questões; efetividade dos teleatendimentos; e efeitos da pandemia a longo prazo, já que a situação ainda não se normalizou e dá sinais de que ainda irá perdurar. Apesar dessas limitações, o estudo refere-se a um público em situação de vulnerabilidade, e por isso entende-se que é relevante para que sejam implementadas medidas de reparação dos problemas.

Para concluir, a pandemia da COVID-19 nos trouxe dor e sofrimento, provocado pelas mudanças, pela falta de estabilidade (Verztman \& Dias, 2020). Como pesquisadores preocupados com a situação de vulnerabilidade de grupos como das crianças e adolescentes com autismo, a situação provocada pela pandemia nos convocou a refletir sobre outras possibilidades de exercício das práticas clínicas, educacionais, sociais e familiares, e a utilizar a pesquisa para elucidar caminhos que contribuam para a formação de uma comunidade mais inclusiva.

\section{Referências}

Amaral, D. G., \& de Vries, P. J. (2020). COVID-19 and Autism Research: Perspectives from Around the Globe. Autism research: official journal of the International Society for Autism Research, 13(6), 844-869. https://doi. org/10.1002/aur.2329.

Ameis, S. H., Lai, M. C., Mulsant, B. H., \& Szatmari, P. (2020). Coping, fostering resilience, and driving care innovation for autistic people and their families during the COVID-19 pandemic and beyond. Molecular Autism, 11(1), 1-9. https://doi. org/10.1186/s13229-020-00365-y.

American Psychiatric Association (2013). Diagnostic and Statistical Manual of Mental Disorders, Fifth Edition (DSM-5). Arlington, VA. Recuperado em 14 set. 2020 de: <https://www.scielo.br/scielo.php?script=sci_ nlinks\&pid=S01037331201400030074100001\&lng=en>.

Aquino, E. M., Silveira, I. H., Pescarini, J. M., Aquino, R., \& Souza-Filho, J. A. D. (2020). Medidas de distanciamento social no controle da pandemia de COVID-19: potenciais impactos e desafios no Brasil. Ciência \& Saúde Coletiva, 25, 24232446. https://doi.org/10.1590/1413-81232020256.1.10502020. 


\section{ARTIGOS}

Araujo, L. A. S., \& Fernandes, E. M. (2020). O cuidado com pessoas com deficiência em tempos da COVID-19: considerações acerca do tema/Caring for people with disabilities in COVID-19 times: considerations on the theme. Brazilian Journal of Health Review, 3(3), 5469-5480. https://doi.org/10.34119/bjhrv3n3-119.

Barbosa, A., Figueiredo, A., Viegas, M., \& Batista, R. (2020). Os impactos da pandemia Covid-19 na vida das pessoas com Transtorno do Espectro Autista. Revista da Seção Judiciária do Rio de Janeiro, 24(48), 91-105. https://doi. org/10.30749/2177-8337.v24n48p91-105fernaandes.

Brasil (1993). Decreto-lei Orgânica da Assistência Social, $n^{\circ}$ 8.742, de 7 de setembro de 1993. Dispõe sobre a organização da Assistência Social e dá outras providências. Presidência da República, Brasil. Recuperado de: $<$ http://www. planalto.gov.br/ccivil_03/leis/18742compilado.htm>.

Brasil (2007). Decreto-lei $n^{\circ} 6214$ de 26 de setembro de 2007. Regulamenta o Benefício de Prestação Continuada da Assistência Social devido à pessoa deficiente e ao idoso de que trata a Lei $n^{\circ} 9.742$, de 7 de dez. 1993, e a Lei $n^{0}$ 10741, de 1 de out. 2003, e acresce parágrafo ao artigo 162 do Decreto $\mathrm{n}^{\circ} 3048$, de 6 de maio 1999. Presidência da República, Brasil. Recuperado de: $<$ https://www2. camara.leg.br/legin/fed/decret/2007/decreto-6214-26-setembro-2007-560259publicacaooriginal-82934-pe.html>.

Brasil (2020). Portaria $n^{\circ} 467$, de 20 de março do Ministério da Saúde - Brasil. Dispõe, em caráter excepcional e temporário, sobre as ações de Telemedicina, com o objetivo de regulamentar e operacionalizar as medidas de enfrentamento da emergência de saúde pública de importância internacional previstas no art. $3^{\circ}$ da Lei $\mathrm{n}^{\circ}$ 13.979, de 6 de fevereiro de 2020, decorrente da epidemia de COVID-19. Diário da República. Recuperado de: <http://www.in.gov.br/en/web/dou/-/ portaria-n-467- de-20-de-marco-de-2020-249312996>.

Brasil (2021a). Tesouro Nacional Transparente do Brasil - Ministério da Economia. Recuperado de: <https://www.tesourotransparente.gov.br/visualizacao/painel-demonitoramentos-dos-gastos-com-covid-19>.

Brasil (2021b). Monitoramento dos Gastos da União com Combate à COVID-19Tesouro Transparente. Recuperado de: <https://www.tesourotransparente.gov.br/ visualizacao/painel-de-monitoramentos-dos-gastos-com-covid-19>.

Brasil (2021c). Painel Coronavirus. Ministério da Saúde. Recuperado de: <https:// covid.saude.gov.br/>.

Briet, G., Sourn-Bissaoui, S. L., Maner-Idrissi, G. L., Peri, M., Marec, O. L., \& Seveno, T. (2017). Longitudinal assessment of children with autism spectrum disorders receiving intensive educational intervention in mainstream school setting. 17th International ESCAP Congress, Genève, Switzerland. Recuperado em 14 set. 2020 de: <https://hal.archives-ouvertes.fr/hal-01728970/>. 
Cahapay, M. B. (2020). How Filipino parents home educate their children with autism during COVID-19 period. International Journal of Developmental Disabilities, 1-4. https://doi.org/10.1080/20473869.2020.1780554.

Cjevic, R., Arnold, S., Foley, K-R., \& Trollor, J. (2018). Neuropsychiatric profile and psychotropic medication use in adults with autism spectrum disorder: Results from the Australian Longitudinal Study of Adults with Autism. BJPsych Open, 4(6), 461-466. https://doi.org/10.1192/bjo.2018.64.

Colizzi, M., Sironi, E., Antonini, F., Ciceri, M., Bovo, C., \& Zoccante L. (2020). Psychosocial and behavioral impact of COVID-19 in autism spectrum disorder: an online parent survey. Brain Sci. 10(6), 341. https://doi.org/10.3390/ brainsci10060341.

Conselho Federal de Medicina (2020). Esclarecimento do CFM sobre a lei da telemedicina - 25 de abril de 2020. Conselho Federal de Medicina. Recuperado de: <http://portal.cfm.org.br/images/PDF/notacfmhonorarioscovid19.pdf>.

Conselho Federal de Psicologia. Resolução $n^{\circ} 4$, de 26 de março de 2020. Dispõe sobre regulamentação de serviços psicológicos prestados por meio de Tecnologia da Informação e da Comunicação durante a pandemia do COVID-19. Conselho Federal de Psicologia. Recuperado de: <https://pesquisa.in.gov.br/imprensa/jsp/ visualiza/index.jsp?data=30/03/2020\&jornal=515\&pagina $=251>$.

Cunha, C., Santos, R. G. H., \& Carlos, D. M. (2016). O filho com transtorno global do desenvolvimento: percepções de mães acerca de cuidados cotidianos. Revista Família, Ciclos de vida e Saúde no Contexto Social. 4(2), 98-106. Recuperado em 10 set. 2020 de: <https://doi.org/10.18554/refacs.v4i2.1646>.

Degli Espinosa, F., Metko, A., Raimondi, M., Impenna, M., \& Scognamiglio, E. (2020). A model of support for families of children with autism living in the COVID-19 lockdown: Lessons from Italy. Behavior Analysis in Practice, 13, 550-558. https://doi.org/10.1007/s40617-020-00438-7.

Dias, B. C., Marcon, S. S., Reis, P., Lino, I. G. T., Okido, A. C. C., Ichisato, S. M. T., \& Neves, E. T. (2020). Dinâmica familiar e rede social de famílias de crianças com necessidades especiais de cuidados complexos/contínuos. Revista Gaúcha de Enfermagem, 41, e20190178. https://doi.org/10.1590/1983-1447.2020.20190178.

Ersoy, K., Altın, B., Bayram Sarıkaya, B., \& Güngörmüş Özkardaş, O. (2020). The comparison of impact of health anxiety on dispositional hope and psychological well-being of mothers who have children diagnosed with autism and mothers who have normal children, in Covid-19 Pandemic. Sosyal Bilimler Araştırma Dergisi, 9(2), 117-126. Recuperado de: <https://dergipark.org.tr/tr/pub/ssrj/ issue $/ 54392 / 737630>$.

Eshraghi A. A., Li C., Alessandri M., Messinger D. S., Eshraghi R. S., Mittal R., \& Armstrong F. D. (2020). COVID-19: overcoming the challenges faced by 


\section{ARTIGOS}

individuals with autism and their families. The Lancet Psychiatry., 7(6), 481-483. https://doi.org/10.1016/s2215-0366(20)30197-8.

Farias, M. N., \& Leite Júnior, J. D. (2020). Vulnerabilidade social e Covid-19: considerações a partir da terapia ocupacional social. Cadernos Brasileiros de Terapia Ocupacional/Brazilian Journal of Occupational Therapy. https://doi. org/10.1590/SciELOPreprints.494.

Fernandes, A. D. S. A., Speranza, M., Mazak, M. S. R., Gasparini, D. A., \& Cid, M. F. B. (2020). Desafios cotidianos e possibilidades de cuidado às crianças e adolescentes com Transtorno do Espectro Autista (TEA) frente à COVID-19. Cadernos Brasileiros de Terapia Ocupacional/Brazilian Journal of Occupational Therapy. https://doi.org/10.1590/SciELOPreprints.955.

Guidotti, M., Gateau, A., Malvy, J., \& Bonnet-Brilhault, F. (2020). Does autism protect against COVID quarantine effects?. medRxiv, 1-8. https://doi.org/10.110 1/2020.10.13.20212118.

Halim, A., Ford, G., Liu-Mayo, S., Glover, E., \& Wall, D. (2020). Multi-modular AI Approach to Streamline Autism Diagnosis in Young Children. Scientific Reports, 10, 5014. https://doi.org/10.1038/s41598-020-61213-W.

Houring, J.den. (2020). Stepping out of isolation: Autistic people and COVID-19. Autism in Adulthood, 2(2), 103-105. http://doi.org/10.1089/aut.2020.29012.jdh.

Instituto Brasileiro de Geografia e Estatística - IBGE (2018). Pesquisa Nacional por Amostra de Domicílios Contínua - PNAD Contínua IBGE. Recuperado de: $<$ https://www.ibge.gov.br/estatisticas/sociais/trabalho/17270-pnad-continua. $\mathrm{html} ?=\& \mathrm{t}=0$-que-e $>$.

Kawabe, K., Hosokawa, R., Nakachi, K., Yoshino, A., Horiuchi, F., \& Ueno, S. I. (2020). Making a brochure about coronavirus disease (COVID-19) for children with autism spectrum disorder and their family members. Psychiatry and clinical neurosciences, 10.1111/pcn.13090. Advance online publication. https://doi. org/10.1111/pen.13090.

Knutsen, J., Crossman, M., Perrin, J., Shui, A., \& Kuhlthau, K. (2019). Sex differences in restricted repetitive behaviors and interests in children with autism spectrum disorder: An Autism Treatment Network study. Autism: the international journal of research and practice, 23(4), 858-868. https://doi. org/10.1177/1362361318786490.

Laboratório de Pesquisa em Saúde Mental e Terapia Ocupacional da UFSCAR (2020). Cuidando da sua saúde mental em tempos de Coronavirus. Recuperado de: $<\mathrm{http}: / /$ www.unifap.br/wp-content/uploads/2020/03/coronavirus_saudemental.pdf>.

Leonardo, F. A. M., \& Morais, A. G. L. de. (2017). Família monoparental feminina: a mulher como chefe de família. Revista do Instituto de Políticas Públicas de Marilia, 3(1), 11-22. https://doi.org/10.33027/2447-780X.2017.v3.n1.02.p11. 
Lewnard, J. A., \& Lo, N. C. (2020). Scientific and ethical basis for social-distancing interventions against COVID-19. Lancet Infect Dis, 20(6), 631-633. Recuperado em 10 set. 2020 de: <https://www.ncbi.nlm.nih.gov/pmc/articles/PMC7118670/m Doi: 10.1016 / S1473-3099 (20) 30190-0>.

Lima, R. C. (2020). Distanciamento e isolamento sociais pela Covid-19 no Brasil: impactos na saúde mental. Physis: Revista de Saúde Coletiva, 30(2), e300214. https://doi.org/10.1590/s0103-73312020300214.

Lima, R. C., \& Couto, M. C. V. (2020). Percepções sobre o autismo e experiências de sobrecarga no cuidado cotidiano: Estudo com familiares de CAPSi da Região Metropolitana do Rio de Janeiro. Cadernos Brasileiros de Saúde Mental/Brazilian Journal of Mental Health, 12(31), 217-244. <https://periodicos.ufsc.br/index.php/ cbsm/article/view/69760>.

Lima Reis, D. D., Neder, P. R. B., da Conceição Moraes, M., \& Oliveira, N. M. (2019). Perfil epidemiológico dos pacientes com Transtorno do Espectro Autista do Centro Especializado em Reabilitação. Pará Research Medical Journal, 3(1), 0-0. http://dx.doi.org/10.4322/prmj.2019.015.

Morales-Hidalgo, P., Roigé-Castellví, J., Hernández-Martínez, C., Voltas, N., \& Canals, J. (2018). Prevalence and characteristics of Autism Spectrum Disorder among Spanish school-Age children. Journal of Autism and Developmental Disorders. 48(9), 3176-3190. https://doi.org/10.1007/s10803-018-3581-2.

Narzisi, A. (2020). Handle the Autism Spectrum Condition during Coronavirus (COVID-19) stay at home period: Ten tips for helping parents and caregivers of young children. Brain Sciences, 10(4). https://doi.org/10.3390/brainsci10040207.

Narzisi, A., Posada, M., Barbieri, F., Chericoni, N., Ciuffolini, D., Pinzino, M., Romano, R., Scattoni, M. L., Tancredi, R., Calderoni, S., \& Muratori, F. (2018). Prevalence of Autism Spectrum Disorder in a large Italian catchment area: a school-based population study within the ASDEU project. Epidemiology and psychiatric sciences, 29, e5. https://doi.org/10.1017/S2045796018000483.

Palacio-Ortiz, J. D., Londoño-Herrera, J. P., Nanclares-Márquez, A., Robledo-Rengifo, P., \& Quintero-Cadavid, C. P. (2020). Trastornos psiquiátricos en los niños y adolescentes en tiempo de la pandemia por COVID-19. Revista Colombiana de Psiquiatría, 49(4),279-288. https://doi.org/10.1016/j. rep.2020.05.006.

Rattazzi, A. (2020). Perspectives from Latin America - Huge shifts in many domains, including health, education, work and family life. In D. G. Amaral, \& P. J. de Vries (2020), COVID-19 and Autism Research: Perspectives from Around the Globe. Autism research: official journal of the International Society for Autism Research, 13(6), 852-853. https://doi.org/10.1002/aur.2329.

Rooke, M. I., Pereira-Silva, N. L., Crolman, S. de R., \& Almeida, B. R. (2019). Funcionamento familiar e rede social de apoio: famílias com crianças com 


\section{ARTIGOS}

síndrome de down. Gerais: Revista Interinstitucional de Psicologia, 12(1), 142-158. https://doi.org/10.36298/gerais2019120111.

Santos, A. J. dos., \& Lemes, M. G. N. (2020). O espectro dos autismos e a psicose infantil: uma questão diagnóstica para a psicanálise. Revista Latinoamericana de Psicopatologia Fundamental, 23(2), 175-197. https://doi.org/10.1590/14154714.2020v23n2p175.3.

Verztman, J., \& Romão-Dias, D. (2020). Catástrofe, luto e esperança: o trabalho psicanalítico na pandemia de COVID-19. Revista Latinoamericana de Psicopatologia Fundamental, 23(2), 269-290. https://doi.org/10.1590/14154714.2020v23n2p269.7.

Viana, B. A., Furtado, L. A. R., \& Vieira, C. A. L. (2020). Invenção e estabilização: uma experiência com crianças autistas em dispositivos de Saúde Mental. Revista Latinoamericana de Psicopatologia Fundamental, 23(2), 313-336. https://doi. org/10.1590/1415-4714.2020v23n2p313.9.

Visani, P., \& Rabello, S. (2012). Considerações sobre o diagnóstico precoce na clínica do autismo e das psicoses infantis. Revista Latinoamericana de Psicopatologia Fundamental, 15(2), 293-308. https://doi.org/10.1590/S141547142012000200006.

World Health Organization (2021). From Coronavirus disease (COVID-19) pandemic. Recuperado de: <https://covid19.who.int/>.

Xu, G., Strathearn, L., Liu, B., O’Brien, M., Kopelman, T. G., Zhu, J., Snetselaar, L. G., \& Bao, W. (2019). Prevalence and treatment patterns of autism spectrum Disorder in the United States, 2016. JAMA pediatrics, 173(2), 153-159. https:// doi.org/10.1001/jamapediatrics.2018.4208.

Zornig, S. (2019). L'isolement personnel comme une forme de jouer avec la solitude. Revista Latinoamericana de Psicopatologia Fundamental, 22(4), 768-781. http:// dx.doi.org/10.1590/1415-4714.2019v22n4p768.7.

\section{Resumos}

(Effects of isolation in COVID-19 pandemic on the behavior of autistic children and adolescents)

Because of Covid-19 pandemic, the World Health Organization has declared a state of public health emergency. Isolation has transformed the social relationships of children and adolescents with autism spectrum disorder. This study aimed to analyze the effects of isolation on the behavior of these individuals. This is a descriptive, cross-sectional study, in which parents or guardians answered an online 
questionnaire. The research results pointed out that confinement has accentuated the symptoms of autism. It is concluded that intervention measures for the subjects and their families are necessary.

Key words: Autistic disorder, behavior, COVID-19, social isolation

(Effets de l'isolement pendant la pandémie du COVID-19 sur le comportement d'enfants et d'adolescents autistes)

Dû̀ à la pandémie du Covid-19, l'Organisation Mondiale de la Santé a déclaré l'état d'urgence de santé publique. L'isolement a transformé les relations sociales d'enfants et d'adolescents atteints de troubles du spectre autistique et cette étude vise à analyser les effets de l'isolement sur leur comportement. Il s'agit d'une étude descriptive et transversale dans laquelle les parents ou tuteurs ont répondu à un questionnaire en ligne. Les résultats de la recherche montrent que le confinement accentuait les symptômes de l'autisme. On conclut que des mesures d'intervention auprès des sujets et de leurs familles sont nécessaires.

Mots clés: Trouble autistique, comportement, COVID-19, isolement social

(Efectos del aislamiento en la pandemia de COVID-19 sobre el comportamiento de niños y adolescentes con autismo)

A raíz de la pandemia de la Covid-19, la Organización Mundial de la Salud declaró un estado de emergencia de salud pública. El aislamiento ha transformado las relaciones sociales de niños y adolescentes con trastorno del espectro autista. El objetivo de este estudio fue analizar los efectos del aislamiento sobre el comportamiento de estos sujetos. Se trata de un estudio transversal descriptivo, en el cual los padres o responsables respondieron un cuestionario en línea. Los resultados de la investigación señalaron que el confinamiento acentuó los síntomas del autismo. Se concluye que las medidas de intervención con los sujetos y sus familias son necesarias.

Palabras clave: Trastorno del espectro autístico, comportamiento, COVID-19, aislamiento social 


\section{ARTIGOS}

Citação/Citation: Givigi, R. C. do N., Silva, R. S., Menezes, E. da C., Santana, J. R. S., \& Teixeira, C. M. P. (2021, set.). Efeitos do isolamento na pandemia por COVID-19 no comportamento de crianças e adolescentes com autimso. Revista Latinoamericana de Psicopatologia Fundamental, 24(3), 618-640. http://dx.doi.org/10.1590/1415-4714.2021v24n3p618.8.

Editora/Editor: Profa. Dra. Ana Maria Galdini Raimundo Oda

Submetido/Submitted: 7.10.2020 / 10.7.2020 Revisado/Revised: $21.12 .2020 / 12.21 .2020$

Aceito/Acepted: $11.1 .2021 / 1.11 .2021$

Copyright: (C) 2009 Associação Universitária de Pesquisa em Psicopatologia Fundamental/ University Association for Research in Fundamental Psychopathology. Este é um artigo de livre acesso, que permite uso irrestrito, distribuição e reprodução em qualquer meio, desde que o autor e a fonte sejam citados / This is an open-access article, which permits unrestricted use, distribution, and reproduction in any medium, provided the original authors and sources are credited.

Financiamento/Funding: Este trabalho não recebeu apoio / This work received no funding.

Conflito de interesses/Conflict of interest: Os autores declaram que não há conflito de interesses. / The authors declare that there is no conflict of interest.

Rosana Carla do Nascimento Givigi

Universidade Federal de Sergipe - UFS (Aracaju, SE, Br), Profa. Associada do Departamento de Fonoaudiologia e do Programa de Pós-graduação em Educação - PPGED.

Av. Augusto Franco, 3500, casa 55 - Ponto Novo

49097-670 Aracaju, SE, Br

rosanagivigi@gmail.com

https://orcid.org/0000-0001-6592-0164

Raquel Souza Silva

Universidade Federal de Sergipe - UFS (Aracaju, SE, Br); Doutoranda do Programa de Pós-Graduação em Educação - PPGED.

Rua Maria Fátima Chagas, 400 - Jabotiana

49095-793 Aracaju, SE, Br

fgaraquelsouza@gmail.com

http://orcid.org/0000-0002-6285-0698

Rev. Latinoam. Psicopat. Fund., São Paulo, 24(3), 618-640, set. 2021 


\section{EdÊNIA da Cunha MEnEZes}

Research Scientist, PhD in Physiology Sciences, Emotional Brain Institute, Nathan Kline Institute, Child and Adolescent Psychiatry Department, New York University (New York, United States).

27, south Mary Francis street, Tappan,

New York, NY., USA.

edenia,menezes@nyulangone.org

https://orcid.org/0000-0001-8889-8457

\section{João Rafael Santos Santana}

Universidade Federal de Sergipe - UFS (Aracaju, SE, Br); Pesquisador de Iniciação Científica do curso de Fonoaudiologia.

Estrada da Cabrita, 103, Cond. Ilha dos Açores, Bloco São Jorge, Apto. 406, Bairro Jabotiana 49096-020 Aracaju-SE, Br

rafaelsttn@gmail.com

https://orcid.org/0000-0002-0566-1858

\section{Clayne Mirelle Pereira Teixeira}

Universidade Federal de Sergipe - UFS (Aracaju, SE, Br); Pesquisadora de Iniciação Científica do curso de Fonoaudiologia.

Conjunto Eduardo Gomes, Rua 85, n. 139

49100-000 São Cristóvão, SE, Br

teixeiramirelle@gmail.com

https://orcid.org/0000-0003-3877-5621

This is an open-access article, which permits unrestricted use, distribution, and reproduction in any medium for non-commercial purposes provided the original authors and sources are credited. 